October 15, 2018

\title{
Self-Consistent Theory of Polymerized Membranes
}

\author{
Pierre Le Doussal* \\ Institute for Advanced Study, Princeton NJ 08540 USA \\ and \\ Leo Radzihovsky \\ Lyman Laboratory, Harvard University, Cambridge MA 02138 USA
}

\begin{abstract}
We study $D$-dimensional polymerized membranes embedded in $d$ dimensions using a selfconsistent screening approximation. It is exact for large $d$ to order $1 / d$, for any $d$ to order $\epsilon=4-D$ and for $d=D$. For flat physical membranes $(D=2, d=3)$ it predicts a roughness exponent $\zeta=0.590$. For phantom membranes at the crumpling transition the size exponent is $\nu=0.732$. It yields identical lower critical dimension for the flat phase and crumpling transition $D_{l c}(d)=\frac{2 d}{d+1}\left(D_{l c}=\sqrt{2}\right.$ for codimension 1$)$. For physical membranes with random quenched curvature $\zeta=0.775$ in the new $T=0$ flat phase in good agreement with simulations.
\end{abstract}

PACS: 64.60Fr,05.40,82.65Dp 
There are now several experimental realizations of polymerized or solid-like membranes, such as protein networks of biological membranes ${ }^{1,2}$, polymerized lipid bilayers ${ }^{3}$ and some inorganic surfaces ${ }^{4}$. Unlike linear polymers, two dimensional sheets of molecules with fixed connectivity and non zero shear modulus are predicted to exhibit a flat phase with broken orientational symmetry. Out of plane thermal undulations of solid membranes which induce a non-zero local Gaussian curvature are strongly suppressed because they are accompanied by in-plane shear deformations 5 . As a result, even "phantom" tethered membranes should be flat at low temperatures ${ }^{5,6}$, and exhibit a quite remarkable anomalous elasticity, with wavevector dependent elastic moduli that vanish and a bending rigidity that diverges at long wavelength ${ }^{7}$. Excluded volume interactions, present in physical membranes, further stabilize the flat phase ${ }^{8}$ but are usually assumed to be otherwise irrelevant to describe its long distance properties. Motivated by recent experiments on partially polymerized vesicles ${ }^{3}$, studies of models with quenched in-plane disorder have shown that the flat phase is unstable at $\mathrm{T}=0$ to either local random stresses ${ }^{9}$ or random spontaneous curvature ${ }^{10}$.

Flat membranes of internal dimensionality D and linear size L are characterized by a roughness exponent $\zeta$ such that transverse displacements scale as $L^{\zeta}$. Nelson and Peliti (NP), using a simple one loop self-consistent theory ${ }^{5}$ for $D=2$ which assumes non-vanishing elastic constants, found that phonon-mediated interactions between capillary waves lead to a renormalized bending rigidity $\kappa_{R}(q) \sim q^{-\eta}$ with $\eta=1$. Since $\zeta=(4-D-\eta) / 2$ they predicted $\zeta=1 / 2$ for

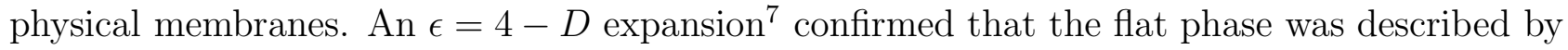
a non trivial fixed point, but with anomalous elastic constants $\lambda(q) \sim \mu(q) \sim q^{\eta_{u}}, \eta_{u}>0$, with $\eta_{u}=4-D-2 \eta$ as a consequence of rotational invariance. Thus, in general $\zeta=\left(4-D+\eta_{u}\right) / 4$ and the NP approximation corresponds to setting $\eta_{u}=0$.

There is presently some uncertainty on the precise value of the roughness exponent for physical membranes. Numerical simulations of tethered surfaces display a range of values for 
$\zeta$ from $0.5^{2}, 0.53^{11}, 0.64^{8,12}$ to $0.70^{13}$. On the other hand, the $O(\epsilon)$ result $^{7}$ suggests a value very close to the NP value $1 / 2,(0.52$ by naively setting $\epsilon=2)$. $\zeta$ should soon be measured from experiments, either directly from light scattering on diluted solutions ${ }^{4}$ or indirectly, from the scale dependence of the elasticity ${ }^{14}$ of lamellar stacks of solid membranes presently under experimental study. The buckling transition ${ }^{6}$, if observed, is controlled by a single exponent related to $\zeta$. It thus seems desirable to explore further possible theoretical predictions for $\zeta$.

In this Letter we introduce a self-consistent approximation which improves on the Nelson Peliti theory ${ }^{5}$ by allowing a non trivial renormalization of the elastic moduli. It is exact in three different limits and compares well with numerical simulations. We construct two coupled selfconsistent equations for the renormalized bending rigidity $\kappa_{R}(q)$ and elastic moduli $\mu_{R}(q), \lambda_{R}(q)$ and solve them in the long wavelength limit. $\kappa_{R}(q)$ is determined by the propagator for the $d_{c}=$ $d-D$ components $\mathbf{h}$ of the out of plane fluctuations $G(q) \sim 1 / q^{4-D-\eta}$ while the elastic moduli are determined by the four-point correlation function of $\mathbf{h}$ fields. Physically, our calculation includes the additional effect of relaxation of in-plane stresses by out of plane displacements. As a result, curvature fluctuations soften elastic constants and screen the phonon-mediated interaction. A similar Self-Consistent Screening Approximation (SCSA) was introduced by Bray $^{15}$ to estimate the $\eta$ exponent of the critical $O(n)$ model (here $d_{c}$ plays the role of the number of components $n$ ) and amounts to a partial resummation of the $1 / d_{c}$ expansion. By construction, the method is exact for large codimension $d_{c}$ to first order in $1 / d_{c}$ and arbitrary $D$. Solving self-consistently then leads to an improved approximation of $\eta\left(d_{c}, D\right)$ (and thus $\zeta$ ) for the small (physical) values of $d_{c}$.

The attractive feature of our theory is that it becomes exact in several other limits. Firstly, because of the Ward identities associated to rotational invariance we find that $\eta\left(d_{c}, D\right)$ is exact to first order in $\epsilon=4-D$ for arbitrary $d_{c}$ and is thus compatible with all presently known results $^{6,7}$. Secondly, for $d_{c}=0$ it gives $\eta=(4-D) / 2$ which is the exact result since clearly 
$\eta_{u}=0$ for $d=D$, and ${ }^{7} \eta_{u}=4-D-2 \eta$. This is at variance with the $O(n)$ model for which the $\mathrm{SCSA}^{15}$ is not exact for $n=0$. Thus we expect this method to give more accurate results for the present problem. Two-loop calculations are in progress ${ }^{16}$ to estimate the deviation. An encouraging indication is the similarity of our method with the remarkably accurate selfconsistent approximation of Kawasaki ${ }^{17}$ for the critical dynamics of the binary fluid mixture, which was shown to be exact to order $\epsilon$, again because of Ward identities, and incorrect to order $\epsilon^{2}$ by a tiny amount. We also apply this method to the crumpling transition of phantom membranes, and to flat membranes with quenched disorder. Details can be found in Ref.16.

In the flat phase, the membrane in-plane and out-of plane displacements are parametrized respectively by a D-component phonon field $u_{\alpha}(x), \alpha=1, . . D$, and a $d_{c}=d-D$ component out-of plane height fluctuations field $\mathbf{h}(x)$. A monomer of internal coordinate $x$ is at position $\mathbf{r}(x)=\left(x_{\alpha}+u_{\alpha}(x)\right) \mathbf{e}_{\alpha}+\mathbf{h}(x)$ where the $\mathbf{e}_{\alpha}$ are a set of D orthonormal vectors. The effective free energy is the sum of a bending energy and an in plane elastic energy (most relevant terms):

$$
F=\int d^{D} x\left[\frac{\kappa}{2}\left(\nabla^{2} \mathbf{h}\right)^{2}+\mu u_{\alpha \beta}^{2}+\frac{\lambda}{2} u_{\alpha \alpha}^{2}\right]
$$

where the strain tensor is $u_{\alpha \beta}=\frac{1}{2}\left(\partial_{\alpha} u_{\beta}+\partial_{\beta} u_{\alpha}+\partial_{\alpha} \mathbf{h} \cdot \partial_{\beta} \mathbf{h}\right)$ To discuss the SCSA in the flat phase it is convenient to first integrate out the phonons ${ }^{1,5}$, and to work with the $d_{c}$-component $\mathbf{h}$ field. In terms of Fourier components the free energy takes the form of a critical theory:

$$
F_{\text {eff }}=\frac{\kappa}{2} \int d k k^{4}|\mathbf{h}(k)|^{2}+\frac{1}{4 d_{c}} \int d k_{1} d k_{2} d k_{3} R_{\alpha \beta, \gamma \delta}(q) k_{1 \alpha} k_{2 \beta} k_{3 \gamma} k_{4 \delta} \mathbf{h}\left(k_{1}\right) \cdot \mathbf{h}\left(k_{2}\right) \mathbf{h}\left(k_{3}\right) \cdot \mathbf{h}\left(k_{4}\right)
$$

with $q=k_{1}+k_{2}$ and $k_{1}+k_{2}+k_{3}+k_{4}=0$ and we use $\int d k$ to denote $\int d^{D} k /(2 \pi)^{D}$. The four-point coupling fourth-order tensor $R(q)$ is transverse to $q$, the longitudinal part having been eliminated through phonon integration. It can be written as $R(q)=b N(q)+\mu M(q)$ with:

$$
N_{\alpha \beta, \gamma \delta}=\frac{1}{D-1} P_{\alpha \beta}^{T} P_{\gamma \delta}^{T} \quad, \quad M_{\alpha \beta, \gamma \delta}=\frac{1}{2}\left(P_{\alpha \gamma}^{T} P_{\beta \delta}^{T}+P_{\alpha \delta}^{T} P_{\beta \gamma}^{T}\right)-N_{\alpha \beta, \gamma \delta}
$$

where $P_{\alpha \beta}^{T}=\delta_{\alpha \beta}-q_{\alpha} q_{\beta} / q^{2}$ is the transverse projector. $\mu$ is the shear modulus and $b=$ $\mu(2 \mu+D \lambda) /(2 \mu+\lambda)$ is proportional to both shear and bulk moduli. The convenience of this 
decomposition is that $M$ and $N$ are mutually orthogonal projectors under tensor multiplication (e.g $M_{\alpha \beta, \gamma \delta} M_{\gamma \delta, \mu \nu}=M_{\alpha \beta, \mu \nu}$ etc...).

We set up two coupled integral equations for the propagator of the $\mathbf{h}$ field and for the renormalized four point interaction. We want to evaluate $<h_{i}(-k) h_{j}(k)>=\delta_{i j} G(k)$ with $G^{-1}(k)=\kappa_{R}(k) k^{4}=\kappa k^{4}+\sigma(k)$ where $\sigma(k)$ is the self energy. The SCSA is defined in diagrammatic form by the graphs of Fig. 1a and 1b, where the double solid line denotes the dressed propagator $G(q)$, the dotted line the bare interaction $R(q)$ and the wiggly line the "screened" interaction $\tilde{R}(q)$ dressed by the vacuum polarization bubbles. We thus obtain two equations, one for $\sigma(k)$ which determines $\eta$, the other for $R$ which determines $\eta_{u}$ :

$$
\begin{gathered}
\sigma(k)=\frac{2}{d_{c}} k_{\alpha} k_{\beta} k_{\gamma} k_{\delta} \int d q \tilde{R}_{\alpha \beta, \gamma \delta}(q) G(k-q) \\
\tilde{R}(q)=R(q)-R(q) \Pi(q) \tilde{R}(q)
\end{gathered}
$$

where $\Pi_{\alpha \beta, \gamma \delta}(q)=\int d p p_{\alpha} p_{\beta} p_{\gamma} p_{\delta} G(p) G(q-p)$ is the vacuum polarization and tensor multiplication is defined above. Because of the transverse projectors, only the component $\Pi(q)_{\text {sym }}$ of $\Pi(q)$ proportional to the fully symmetric tensor $S_{\alpha \beta, \gamma \delta}=\delta_{\alpha \beta} \delta_{\gamma \delta}+\delta_{\alpha \gamma} \delta_{\beta \delta}+\delta_{\alpha \delta} \delta_{\beta \gamma}$ contributes in (4b). Defining $\Pi(q)_{\text {sym }}=I(q) S$, simple algebra gives $\tilde{R}(q)=\tilde{\mu}(q) M+\tilde{b}(q) N$ with renormalized shear and shear-bulk moduli, and the new equations:

$$
\begin{gathered}
\tilde{\mu}(q)=\frac{\mu}{1+2 I(q) \mu} \quad \tilde{b}(q)=\frac{b}{1+(D+1) I(q) b} \\
\sigma(k)=\frac{2}{d_{c}} \int d q \frac{\tilde{b}(q)+(D-2) \tilde{\mu}(q)}{D-1}\left(k P^{T}(q) k\right)^{2} G(k-q)
\end{gathered}
$$

We now solve these equations in the long-wavelength limit. Substituting $G(k) \sim \sigma^{-1}(k) \sim$ $Z / k^{4-\eta}$ in $(5 \mathrm{a}, \mathrm{b})$, with $Z$ a non-universal amplitude, we find that the vacuum polarization integral diverges as:

$$
I(q) \sim Z^{2} A(D, \eta) q^{-\eta_{u}}
$$


where $\eta_{u}=4-D-2 \eta$ is the anomalous exponent of phonons. Substituting in (5a,b), and defining the amplitude: $\int d q q^{\eta_{u}}(k-q)^{-(4-\eta)}\left(k P^{T}(q) k\right)^{2}=B(D, \eta) k^{4-\eta}$, one finds (for $\left.\mu, b>0\right)$ that the $Z$ and $k^{4-\eta}$ factors cancel and that $\eta$ is determined self-consistently by the equation for the amplitude: $d_{c}=\frac{D}{D+1} \frac{B(D, \eta)}{A(D, \eta)}$, which after calculation of the integrals defining A,B gives:

$$
d_{c}=\frac{2}{\eta} D(D-1) \frac{\Gamma\left[1+\frac{1}{2} \eta\right] \Gamma[2-\eta] \Gamma[\eta+D] \Gamma\left[2-\frac{1}{2} \eta\right]}{\Gamma\left[\frac{1}{2} D+\frac{1}{2} \eta\right] \Gamma\left[2-\eta-\frac{1}{2} D\right] \Gamma\left[\eta+\frac{1}{2} D\right] \Gamma\left[\frac{1}{2} D+2-\frac{1}{2} \eta\right]}
$$

For $D=2$ this equation can be simplified, and one finds (Fig. 2):

$$
\eta\left(D=2, d_{c}\right)=\frac{4}{d_{c}+\sqrt{16-2 d_{c}+d_{c}^{2}}}
$$

Thus for physical membranes we obtain: $\eta=0.821, \eta_{u}=0.358$ and:

$$
\zeta=1-\frac{\eta}{2}=\frac{\sqrt{15}-1}{\sqrt{15}+1}=0.590 .
$$

roughly at midvalue of the present numerical simulations. From (5) we also obtain $\underset{q \rightarrow 0}{\operatorname{Lim}} \tilde{\lambda}(q) / \tilde{\mu}(q)=$ $\frac{-2}{D+2}$ (i.e a negative Poisson ratio).

Expanding the result $(7)$ in $1 / d_{c}$ one obtains:

$$
\eta=\frac{8}{d_{c}} \frac{D-1}{D+2} \frac{\Gamma[D]}{\Gamma\left[\frac{D}{2}\right]^{3} \Gamma\left[2-\frac{D}{2}\right]}+O\left(\frac{1}{d_{c}^{2}}\right)=\frac{2}{d_{c}}+O\left(\frac{1}{d_{c}^{2}}\right) \quad(\text { for } D=2)
$$

which coincides with the exact result ${ }^{6,7}$, as expected by construction of the SCSA. Similarly, expanding (7) to first order in $\epsilon=4-D$ one finds:

$$
\eta=\frac{\epsilon}{2+d_{c} / 12}
$$

also in agreement with the exact result ${ }^{6,7}$. This is not a general property of SCSA. Here it can be traced to the vertex and box diagrams of Fig. 1c being convergent. Indeed, because of the transverse projectors in (2-3) one can always extract one power of external momentum from each external $h$ legs, which lowers the degree of divergence from naive power counting. As a result, if one decouples the 4-point vertex $R$ via a mediating field, the only counterterms needed are for two-point functions. 
We have analyzed the crumpling transition of phantom membranes by the same method, applied to the isotropic theory of Ref.18. The exponent $\eta=\eta_{c r}$ at the transition is determined $\operatorname{by}^{16}$ :

$$
d=\frac{D(D+1)(D-4+\eta)(D-4+2 \eta)(2 D-3+2 \eta) \Gamma\left[\frac{1}{2} \eta\right] \Gamma[2-\eta] \Gamma[\eta+D] \Gamma\left[2-\frac{1}{2} \eta\right]}{2(2-\eta)(5-D-2 \eta)(D+\eta-1) \Gamma\left[\frac{1}{2} D+\frac{1}{2} \eta\right] \Gamma\left[2-\eta-\frac{1}{2} D\right] \Gamma\left[\eta+\frac{1}{2} D\right] \Gamma\left[\frac{1}{2} D+2-\frac{1}{2} \eta\right]}
$$

At the transition the radius of gyration scales as $R_{G} \sim L^{\nu}$ with $\nu=\left(4-D-\eta_{c r}\right) / 2$. For $d=3$ and $D=2$ we find $\eta_{c r}=0.535$ and $\nu=0.732$ (Haussdorf dimension $d_{H}=2.73$ ). The embedding dimension $d_{u}(D)$ above which self-avoidance is irrelevant for the membrane at the crumpling transition is determined by the condition $d_{u}=4 D /\left(4-D-\eta_{c r}\left(d_{u}\right)\right)$. Using (12) we find that $d_{u}(2)=4.98$.

The present method gives interesting predictions for lower critical dimensions. In the flat phase, orientational order (i.e in $\nabla \mathbf{h}$ ) disappears for $D<D_{l c}$, where $2-\eta\left(D_{l c}, d_{c}\right)=D_{l c}$. From (7) this is equivalent to $d_{c}=D_{l c}\left(D_{l c}-1\right) /\left(2-D_{l c}\right)$. On the other hand, the lower critical dimension $D_{l c}^{\prime}(d)$ for the crumpling transition is defined by $2-\eta_{c r}\left(D_{l c}^{\prime}, d\right)=D_{l c}^{\prime}$, or equivalently from $(12), d=D_{l c}^{\prime} /\left(2-D_{l c}^{\prime}\right)$. Since $d=D /(2-D)$ is clearly equivalent to $d_{c}=D(D-1) /(2-D)$ we find that the lower critical dimensions of the crumpling transition and of the flat phase, as predicted by SCSA, are identical, and given by $D_{l c}(d)=2 d /(1+d)$. Since they originate from very different calculations, this indicates that the SCSA is quite consistent. For codimension 1 manifolds $D_{l c}=\sqrt{2}$ and for fixed embedding space $d=3, D_{l c}=3 / 2$. $D_{l c}$ increases from $D_{l c}=1$ for $d_{c}=0$, to $D_{l c}=2$ when $d_{c} \rightarrow \infty$ as expected. Note that for $d>3$ self-avoidance cannot modify the above results, while for $d<3$ it is an open question.

We can compare $(8,12)$ with recent simulations ${ }^{19}$ of $D=2$ membranes with self-avoidance in higher $d_{c}$. The membranes are found flat in $d=3,4$ with $\zeta(d=3)=0.64 \pm 0.04, \zeta(d=4)=$ $0.77 \pm 0.04$, whereas we obtain $0.59,0.67$, respectively. The membrane is crumpled in $d=5$ 
with $\nu=0.8 \pm 0.06$, although $d=5$ seems almost marginal, whereas we find $\nu=0.8$ at the crumpling transition where self avoidance is irrelevant, although almost marginally so.

Flat membranes with random spontaneous curvature are described by adding the term $-\int d^{D} x \mathbf{c}(x) \cdot \nabla^{2} \mathbf{h}(x)$ in the energy (1), where $\mathbf{c}(\mathrm{x})$ are Gaussian quenched random variables ${ }^{10}$. Within a replica symmetric SCSA, we find a marginally unstable $T=0$ fixed point, i.e a long-wavelength solution only if $T \rightarrow 0$ first. Defining the replica connected and off-diagonal exponents $\eta, \eta^{\prime}$, by $\overline{<\mathbf{h}(-q) \mathbf{h}(q)>_{c}} \sim q^{-(4-\eta)}, \overline{\langle\mathbf{h}(-q) \mathbf{h}(q)>} \sim q^{-\left(4-\eta^{\prime}\right)}$ we find ${ }^{16}$ at this fixed point: $\eta^{\prime}=\eta, \eta\left(d_{c}, D\right)=\eta_{\text {pure }}\left(4 d_{c}, D\right)$. Thus one can simply replace $d_{c}$ in the pure result by $4 d_{c}$ ! Again this agrees with the $1 / d_{c}$ and $\epsilon$ expansions ${ }^{10}$. For physical membranes $D=2$, $d_{c}=1$, we find from $(8)$ :

$$
\eta=2 /(2+\sqrt{6})=0.449 \quad \zeta=0.775
$$

comparing well with the numerical simulation ${ }^{10}$ result $\zeta=0.81 \pm 0.03$. By analogy with the random field problem ${ }^{20}$, it is quite possible that the equality $\eta=\eta^{\prime}$, conjectured in Ref. 10 to all orders, be corrected when replica-symmetry breaking is included.

In conclusion, we have presented a self-consistent theory of polymerized membranes which becomes exact in three limits (large $d_{c}$, small $\epsilon=4-D$, and $d_{c}=0$ ). By construction, it satisfies the exponent relations $\eta_{u}=4-D-2 \eta$ and $^{16} 1 / \nu^{\prime}=D-2+\eta$. These relations are exact in the true theory because of rotational invariance ${ }^{6,7}$. It thus predicts $\nu^{\prime}=1.218$ and $\delta^{\prime}=1.436$ for the buckling transition exponents ${ }^{6}$. It contradicts the conjecture ${ }^{2} \zeta=1 / 2$.

We thank D. Nelson, M. Mezard for discussions. PLD acknowledge support from NSF grant DMS-9100383 and LR from the Hertz Graduate Fellowship. 


\section{References}

*Also LPTENS, Ecole Normale Superieure, 24 rue Lhomond, Paris 75231 Cedex 05, Laboratoire Propre du CNRS.

1. See, e.g, Statistical Mechanics of Membranes and Interfaces, edited by D.R. Nelson, T. Piran, S. Weinberg ( World Scientific, Singapore 1988 ), and S. Leibler in Proceedings of the Cargese school on biologically inspired physics, (1990), to be published.

2. R. Lipowsky and M. Girardet, Phys. Rev. Lett. 652893 (1990).

3. M. Mutz, D. Bensimon, M.J. Brienne, Phys. Rev. Lett. 67923 (1991).

4. X. Wen et al. Nature 355, 426 (1992)

5. D. R. Nelson and L. Peliti, J. Phys. (Paris) 48, 1085 (1987).

6. F. David and E. Guitter, Europhys. Lett. 5, 709 (1988). E. Guitter, F. David, S. Leibler and L. Peliti, J. Phys. France 501787 (1989).

7. J.A. Aronovitz and T.C. Lubensky, Phys. Rev. Lett. 60, 2634 (1988), J.A. Aronovitz, L. Golubovic, T.C. Lubensky, J. Phys. France 50609 (1989).

8. F.F. Abraham, D.R. Nelson, J. Phys. France 512653 (1990). F.F. Abraham, W.E. Rudge and M. Plishke, Phys. Rev. Lett. 62, 1757 (1989).

9. D.R. Nelson, L. Radzihovsky, Europhys. Lett. 16, 79 (1991), L. Radzihovsky, P. Le Doussal, J. Phys. I France 2, 599 (1992).

10. D.C. Morse, T.C. Lubensky, G.S. Grest, Phys. Rev A 45 R2151 (1992). Morse Lubensky, Preprint 1992. 
11. F. Abrahams, Phys. Rev. Lett. 67, 1669 (1991).

12. S. Leibler and A. Maggs, Phys. Rev. Lett. 63, 406 (1989).

13. G. Gompper and D.M. Kroll, J. Phys. I France 2, 663 (1992). (1992).

14. J. Toner, Phys. Rev. Lett. 641741 (1990).

15. A.J. Bray, Phys. Rev. Lett. 32, 1413 (1974).

16. P. Le Doussal, L. Radzihovsky, to be published.

17. E.D. Siggia, B.I. Halperin and P.C. Hohenberg, Phys. Rev. B 132110 (1976).

18. M. Paczuski, M. Kardar and D.R. Nelson, Phys. Rev. Lett. 60, 2638 (1988).

19. G. Grest, J. Phys. I France 1,1695 (1991).

20. M. Mezard, A.P. Young, Preprint LPTENS 92/2 


\section{FIGURE CAPTIONS}

Figure 1 : graphical representation of the SCSA: (a) self energy, (b) interaction. (c) UV finite vertex and box diagrams.

Figure $2: \zeta$ as a function of $d$ for two-dimensional membranes $D=2$. Solid curve: SCSA result (8). Dashed dotted curve: $O(\epsilon)$ result, setting $\epsilon=2$. Dashed curve: corresponds to $\eta=2 / d$ chosen (somewhat arbitrarily) in Ref. 6 as a possible interpolation to finite $d$ (asymptotic to the solid curve for $d \rightarrow \infty$ ). 